\title{
A Multi-Zoned Self-Resistance Electric Heating Method for Curing Irregular Fiber Reinforced Composite Parts
}

\author{
Shuting Liu ${ }^{\mathrm{a}, 1}$, Yingguang Li ${ }^{\mathrm{a}}$, Yingxiang Shen ${ }^{\mathrm{a}}$ and Yee Mey Goh ${ }^{\mathrm{b}}$ \\ ${ }^{a}$ College of Mechanical and Electrical Engineering, Nanjing University of Aeronautics \\ and Astronautics, Nanjing,210001, China \\ ${ }^{\mathrm{b}}$ School of Mechanical, Electrical and Manufacturing Engineering, Loughborough \\ University, Leicestershire, LE11 3TU, UK
}

\begin{abstract}
Fibre Reinforced Polymers (FRP) have been widely applied in various industries due to their outstanding properties. As a promising curing technology for FRP parts, the self-resistance electric (SRE) heating method has attracted plenty of attention. However, it is difficult for the SRE heating method to uniformly cure the FRP parts with irregular structures. In this paper, a multi-zoned SRE heating method is proposed, in which the FRP part is divided into several heating zones and the temperature of each zone is regulated independently. A multi-channel electrical voltage control system is developed to realise the multi-zoned SRE heating of a wing-shaped FRP part, in which a rapid zone-based temperature control responsiveness is achieved, and the maximum temperature difference is reduced from $60{ }^{\circ} \mathrm{C}$ to less than $10{ }^{\circ} \mathrm{C}$, reaching $2.5{ }^{\circ} \mathrm{C}$ at its best. This work presents an alternative for the high efficiency and energy-saving curing process of FRP parts.
\end{abstract}

Keywords. Reinforced polymer, Curing, Self-resistance electric heating

\section{Introduction}

In recent years, Fibre-Reinforced Polymer (FRP) composite has seen rapid growth in multiple domains such as aerospace, automotive, and energy industries due to their outstanding mechanical and structural properties [1]. As one typical type of the FRP composite that has excellent electric or electromagnetic characteristics [2], Glass/Carbon Hybrid Reinforced Polymers (G/CHFRP) is drawing increased interest for the applications of sensing, electromagnetic interference, structural batteries, and low-cost load-bearing. The manufacturing process of FRP composites especially related to the curing process requires a high-temperature heating treatment to enable the matrix resin to undergo a chemical or physical evolution process, resulting in final components that both possess geometric accuracy and load-bearing strength. An emerging curing technology called Out-of-Autoclave (OoA) method presents a high efficiency, energysaving, and low-cost alternative to the traditional autoclave curing process which comes with a high price in terms of capital expense, energy consumption, and cycling time [3].

\footnotetext{
${ }^{1}$ Corresponding Author, Yingguang Li, College of Mechanical and Electrical Engineering, Nanjing University of Aeronautics and Astronautics, Nanjing, China; E-mail: liyingguang@nuaa.edu.cn
} 
More recently, OoA processing has been successfully employed in the manufacture of FRP parts for unmanned spacecraft and commercial passenger aircraft.

As a promising OoA curing technology, Self-Resistance Electric (SRE) heating method is promising owing to its superior energy efficiency and low capital investment [4]. In the SRE heating process, the electrical current is directly fed into the conductor (carbon fibre) by using edge-contacted electrodes, and then a quick-responded Joule heat is geneated. This method exhibits many advantages such as absolute volumetric heating, rapid heating rate, and high energy utilisation ratio. However, despite these merits, a inherent defect has been limiting its wide industrialised applications. Because the electrical current density is highly dependent on the cross-sectional area of the part, the global self-generated Joule heat is unable to uniformly heat the composite part with an irregular geometric structure.

From previous literature, several researchers have investigated thermal responsiveness, temperature field, and mechanical performance of cured samples of the SRE heating process. Only a few studies have reported the influence of geometrical shape on heating uniformity. Athanasopoulos et al [5] investigated the temperature fields of the hat-shaped FRP parts that being directly conducted by integral electrodes, where the temperature uniformity was evaluated. In the field of metal sheet thermal forming, Lackmann et al [6] proposed that using a complemental element brought into contact under the part with irregular section to obtain uniform cross-section that could generate even Joule heat distribution. Behrens and Hübner [7] presented a similar technology that utilises the external resistance element to convert the original non-rectangular shape into several rectangles. Demazel et al [8] reported a further methodology, which applied the minimum complemental material to form rectangles, and each rectangle was controlled by an individual power source that was connected with the corresponding electrode pair. The above research provided inspiring research ideas for the uniform SRE heating of FRP irregular parts. However, as shown in Figure 1 (a), (c) and (e), when the electric isotropic material is conducted by multiple independent narrow electrodes, transverse charge transfer will occur between adjacent zone with unequal potential, and directly lead to a large amount of energy loss as well as the local over-hot spot. Therefore, existing methods may not be directly applicable to the SRE heating of the FRP composite part without considering the effect of the electric anisotropy.
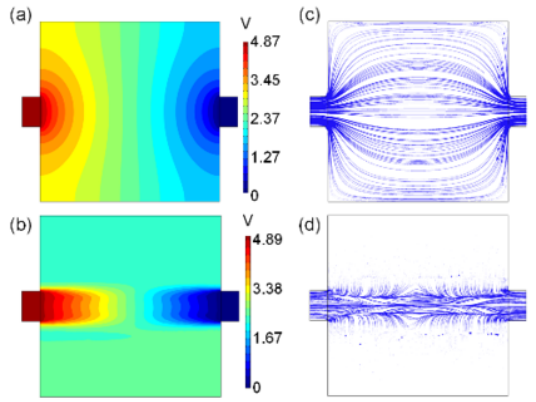
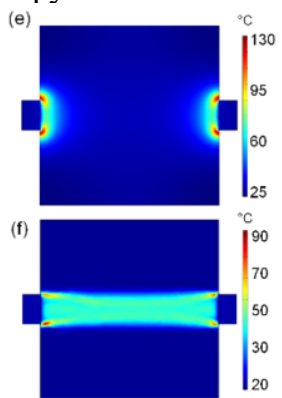

Figure 1. Electrical and Joule heat properties of anisotropic and isotropic material (a) \& (b): electric field intensity field, (c) \& (d): current line, (e) \& (f): temperature field

In this paper, we developed a multi-zoned SRE heating methodology that could achieve a well-defined stripe of Joule heat in FRP laminates and further uniformly heat the irregular parts by applying multiple independent zones. As shown in Figure 1 (b), (d) and (f), by embedding the electrode sheets in specific layers and areas, the material 
between the two electrodes exhibits high electrical anisotropy, which restrains the electric current from spreading through the transverse direction and generate relative uniform temperature distribution. A typical wing-shaped G/CHFRP structure is cured by using a multi-zoned SRE heating method using a self-developed single power multichannel voltage control system. The zoned temperature control responsiveness is analysed and the temperature distribution fields of the wing-shaped part by multi-zoned SRE method are demonstrated and discussed.

\section{Experiments}

\subsection{Laying-up sequence and bagging arrangement}

A typical wing-shaped FRP part that has an uneven conductive length is chosen to validate the effectiveness of the presented zoned SRE method. In Figure 2 (a), the dimension data of the part is illustrated. Generally, for carbon FRP composite, the electrical conductivity along fibre axial direction is 1,000 times higher than that of perpendicular-to-fiber direction, and this magnitude of electrical anisotropy is enough for confining current to a particular area. In this paper, to reinforce the electrical anisotropy, a G/CHFRP laminate is adopted, in which each of the heated carbon fiber layers is laid between two glass fiber prepregs, as shown in Figure 2 (b). Cyclic symmetric layering sequence $\left[0^{\circ} / 45^{\circ} / 90^{\circ} /-45^{\circ}\right] 2 \mathrm{~s}$ is applied, and there are two heating layers $\left(90^{\circ}\right.$ carbon fiber) inside the laminate.
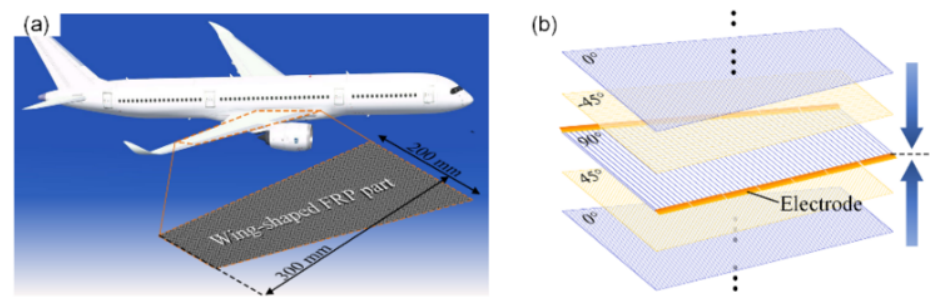

Figure 2. Schematic of wing-shaped part. (a): dimensions and (b): lay-up sequence.

Red copper thin sheet $(0.1 \mathrm{~mm})$ is utilised as electrode layers, which are overlapped by $5 \mathrm{~mm}$ with the FRP layer. In the multi-zoned SRE heating process, each zone has a width of $40 \mathrm{~mm}$, and the cables are soldered by tin with the copper sheets, as shown in Figure 3(a). The whole material system is encapsulated in a vacuum bag following the standard procedure of the FRP curing process (see Figure 3 (b) and (c)).
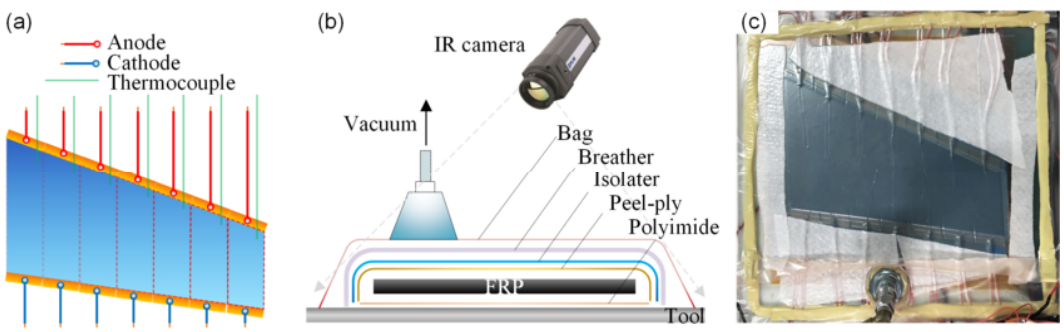

Figure 3. Sketch of curing arrangement. (a): electrodes, sensors setup and (b):Vacuum bagging arrangement. (c): Experimental photograph. 


\subsection{Temperature monitoring}

Two different temperature monitoring methods respectively: thermocouple and infrared (IR) camera are applied during SRE heating processes. As shown in Figure 3 (a), seven thermocouples are bonded at the edge area $(3 \mathrm{~cm}$ inside) of 7 zones using polyimide tape. Their feedback signals are displayed, recorded, and used as the input data of the PID temperature control meter.

\subsection{Multi-channel power control system}

Multi-zoned SRE heating requires independent power control for different areas. The previous research [8] adopted a multiple power supply system to achieve differential heating of diverse rectangular areas. Here a single power multi-channel voltage control system is used. As shown in Figure 4, in this system, only one PID control meter is used, which monitors the input signal of the average temperature value computed from the data of all thermocouple sensors. A Metal-Oxide-Semiconductor Field-Effect Transistor (MOSFET) network is constructed, whose output is a duty factor matrix controlled by CPU according to the temperature pattern monitored by thermocouple sensors. Thus, by using this highly integrated system, the voltage of each zone can be regulated independently to achieve the homogeneous temperature distribution.

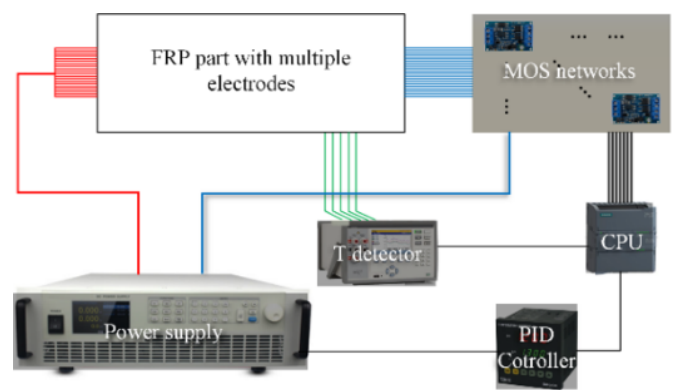

Figure 4. Layout of single power multi-channel voltage control system.

\section{Discussion}

\subsection{Zoned temperature control responsiveness}

As shown in Figure 5, first of all, by embedding electrodes in a specific layer, the current is prevented to diffuse through-thickness or along the perpendicular-to-the fibre direction in the 2D plane, which directly results in well-defined Joule heat stripes between every electrode pair and there are no edge-concentration hotpots. This can be attributed to that the current selectively passes through the direction with minimum resistance. Secondly, as time goes on, driven by the MOSFET network, different thermal patterns (along Xdirection) such as linear, parabola, sine, impulse wave, and high-low double platform can be rapidly switched. This indicates that by controlling the output of the MOSFET network, Joule heat can be coded as any thermal pattern along a certain direction in FRP composites, to fit in various complex geometric profiles. 


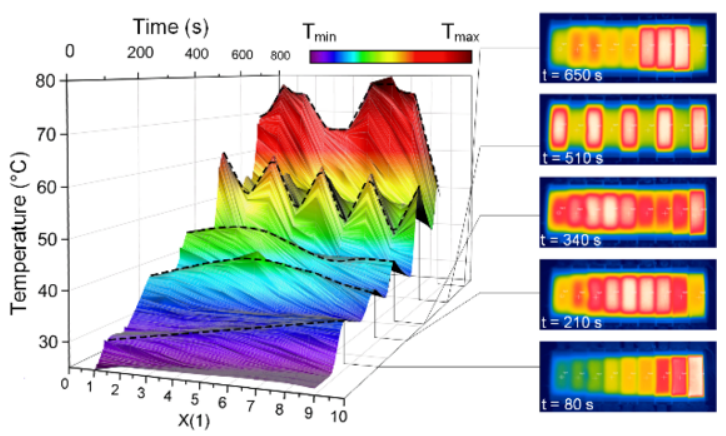

Figure 5. Rapid regulation of zoned temperature patterns over time.

\subsection{Comparison on temperature fields}

The temperature fields of traditional integral SRE heating and multi-zoned SRE heating are compared in this section. As shown in Figure 6 (a) and (b), due to the current density is dependent on the conductive length, the shorter heating segment has the higher temperature. The maximum in-plane temperature difference is over $60{ }^{\circ} \mathrm{C}$ during the dwelling stage, which produces a low temperature to the wing root area so that the crosslinking reaction cannot be initiated. In the multi-zoned SRE heating process, driven by the MOSFET network, 7 independent zones respectively generate appropriate energy according to the feedback of the temperature sensors. The maximum temperature difference is below $10{ }^{\circ} \mathrm{C}$ and remained below $5{ }^{\circ} \mathrm{C}$ for most of the time. It can be seen in the IR images of Figure $6(\mathrm{~d})$, the whole wing-shaped part maintains a high temperature uniformity. The zoned SRE cured part is shown in Figure 7 (a).

Figure 7 (b) shows the change in duty factors of the MOSFET network. The initial duty factors are calculated based on the relationship between the conductive length and the Joule heat power, which are $1,0.92,0.84,0.76,0.68,0.60$, and 0.51 respectively from the wing root to wingtip. As the reaction goes on, because the electrical conductivity of the materials in each zone changes as the temperature rises and crosslinking reaction occurs, the duty factor of each zone is regulated accordingly.
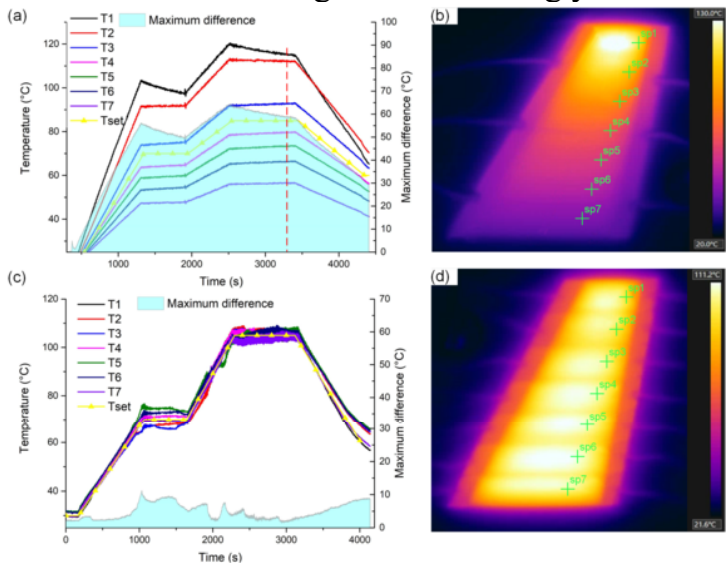

Figure 6. Temperature field results. (a), (c) Temperature data and (d), (d) IR images of integral SRE (top row) and zoned SRE (bottom row) heating process. 

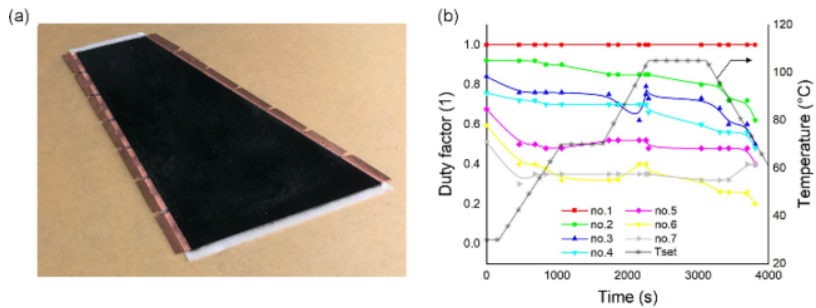

Figure 7. Finished part and duty factor curves. (a) Zoned SRE cured wing-shaped part, and (b) duty factors change during zoned heating process.

\section{Conclusion}

This paper has presented a multi-zoned SRE heating method for irregular FRP parts. By considering the electrical anisotropy of FRP composites, embedding the electrodes sheet into specific layers and areas, well-defined Joule heat stripe is achieved. A multi-channel electrical voltage control system is developed. During the whole multi-zoned SRE heating process of a wing-shaped FRP part, the maximum temperature difference is reduced from $60{ }^{\circ} \mathrm{C}$ of integral SRE to less than $10{ }^{\circ} \mathrm{C}$, reaching $2.5^{\circ} \mathrm{C}$ at its best in the late dwell stage. The present work provides a potential solution for the uniform SRE heating of irregular FRP parts.

\section{Acknowledgements}

This work was supported by National Natural Science Foundation of China (Grant no. 51775261) and Postgraduate Research\&Practice Innovation Program of Government of Jiangsu Province (KYCX18_0319), the authors sincerely appreciate the continuous support provided by our industrial collaborators.

\section{References}

[1] Hassan MH, Othman AR, Kamaruddin S, A review on the manufacturing defects of complex-shaped laminate in aircraft composite structures. Int. J. Adv. Manuf. Technol. 2017 Feb; 91(9): 4081-4094.

[2] Forintos $\mathrm{N}$ and Czigany T, Multifunctional application of carbon fiber reinforced polymer composites: Electrical properties of the reinforcing carbon fibers- A short review. Compos. B. Eng. 2019 Apr;162: 331-343.

[3] Witik RA, Gaille F, Teuscher R, Economic and environmental assessment of alternative production methods for composite aircraft components. J. Clean. Prod. 2012 Mar; 29: 91-102.

[4] Liu ST, Li YG, Shen Y, Mechanical performance of carbon fiber/epoxy composites cured by selfresistance electric heating method. Int. J. Adv. Manuf. Technol. 2019 May;103.1032.

[5] Athanasopoulos N, Kostopoulos G, Vlacos D, Kostopoulos V, Temperature uniformity analysis and development of open lightweight composite molds using carbon fibers as heating elements. Compos. B. Eng. 2013 Mar; 50: 279-289.

[6] Lackmann J, Konrad S, Lambers H, Huschen U, Heating device for conductive heating of a sheet metal blank. U.S. Patent Application, 14/671,363 (2015).

[7] Behrens BA, Hübner S, Method for conductively heating sheet metal, and heating device for carrying out said method. Patent WO2015124604A1 (2015 10).

[8] Demazel N, Laurent H, Carin M, A direct resistance heating method for shaped blank. J Manuf Process. 2021 Dec; 62: 772-783. 